\section{L'article scientifique en sciences de l'éducation : un genre textuel à l'apparence uniforme constitué de sections aux
caractéristiques distinctes} Christiane Blaser Université de Sherbrooke (Canada) Judith Emery-Bruneau Université du Québec en Outaouais (Canada) Stéphanie Lanctôt Université de Sherbrooke (Canada)
Scientific article in educational sciences: A textual genre with a

uniform appearance composed of sections with distinct characteristics

\section{ésumé}

L'article scientifique est un genre textuel omniprésent dans le milieu universitaire, mais il prend des formes variables selon les champs disciplinaires. Quelles sont donc les caractéristiques de l'article scientifique, rédigé en français, dans le domaine des sciences de l'éducation? Pour le savoir, nous avons analysé 70 articles tirés de sept revues scientifiques canadiennes pour en dégager, section par section, les caractéristiques communicationnelles, textuelles, sémantiques, grammaticales et visuelles. II ressort que certaines caractéristiques varient d'une section à l'autre et qu'il serait donc fécond, dans un objectif didactique, d'aborder

I'article scientifique comme la somme de ses parties plutôt que comme un bloc monolithique.

\section{Mots-clés}

Genre textuel, littératie universitaire, article scientifique, rapport à l'écrit, analyse textuelle.

\section{Abstract}

The scientific article is a textual genre that is omnipresent in the academic community, which can take various forms according to the disciplinary field. Considering this, what are the characteristics of the scientific article in the domain of sciences of education? In order to answer the question, we analyzed seventy articles in French drawn from six Canadian scientific journals, focusing specifically on their communicational, textual, semantic, grammatical, and visual characteristics.

It appears that some of these characteristics vary from one section to another. As such, it is therefore important when the objective is didactic to approach the scientific article like the sum of its parts rather than a monolithic block.

Keywords

Textual genre, academic literacy, scientific article, relation to writing, text analysis.
Omniprésent dans le milieu universitaire, l'article scientifique joue un rôle majeur dans la construction et la diffusion des savoirs scientifiques. Pourtant, même si les étudiantes et étudiants des cycles supérieurs sont encouragés très tôt dans leur parcours de maitrise ou de doctorat à publier les résultats ou l'état d'avancement de leurs travaux de recherche dans des revues, l'article scientifique ne fait pas systématiquement l'objet d'un enseignement dans la formation universitaire, pas plus d'ailleurs que les autres écrits qui jalonnent la formation à la recherche. Or, tout comme n'importe quel genre de texte, l'article scientifique comporte ses propres caractéristiques, lesquelles, de plus, sont spécifiques à chaque domaine d'études : les chercheurs et chercheuses en biologie par exemple ne rédigent pas leurs articles scientifiques en épousant exactement la même structure ou en recourant au même mode de discours que leurs collègues des sciences de l'éducation, de lettres ou d'autres domaines scientifiques.

Si certaines universités offrent, dans les programmes d'études supérieures, un cours à option consacré à l'écrit ${ }^{1}$, dans d'autres institutions, les directions de programmes tiennent pour acquis que la responsabilité de l'encadrement des écrits relève essentiellement des ressources professorales qui supervisent les étudiantes et étudiants. Pourtant, depuis quelques années, dans le champ de la didactique de l'écrit et, plus récemment, dans le champ des littératies universitaires, il est admis que l'apprentissage de l'écrit n'a pas de fin et devrait donc aussi se poursuivre à l'université (Blaser et Lanctôt, 2021; Boch, 2013; Delcambre et Lahanier-Reuter, 2010, 2012; Delcambre et Pollet, 2014; Donahue, 2008, 2010; 2021; Frier, 2015; Pollet, 2001, 2004; Thyrion, 2011). Ce constat repose, d'une part, sur le fait que chaque nouveau palier de la scolarité présente des défis spécifiques sur le plan des écrits à lire et à produire et, d'autre part, que chaque discipline scolaire ou universitaire a des genres textuels qui lui sont propres. De nombreux étudiants, surtout dans un contexte de massification 
de l'enseignement supérieur, éprouvent des difficultés à réaliser des travaux de qualité parce qu'ils ne sont pas outillés pour identifier et comprendre les caractéristiques des genres de textes demandés; ils ont donc besoin de soutien dans leur démarche d'acculturation aux discours universitaires, durant tout leur parcours, du premier au troisième cycle (Pollet, 2001; Pollet et al., 2010; Scheepers et al., 2021). Ce soutien peut prendre diverses formes et provenir de sources variées comme des activités créditées, ou non, portant sur l'écriture universitaire (Boch, Cavalla, Pétillon et Rinck, 2015; Lafont-Terranova, Niwese et Colin, 2016; Pollet, 2014), la consultation de guides de rédaction (Belleville, 2014; Jutras, 2019) ou autres articles sur le sujet.

Dans cet article, nous présentons la démarche et quelques résultats d'une recherche visant à décrire les caractéristiques de l'article scientifique dans le domaine des sciences de l'éducation. Pour mener à bien ce projet, nous avons analysé, section par section ${ }^{2}, 70$ articles de ce domaine pour dégager à la fois les caractéristiques communes à l'ensemble des articles et les caractéristiques spécifiques à chacune des sections des articles. Ce projet a pour objectif ultime l'amélioration des connaissances au sujet du genre textuel qu'est l'article scientifique dans le but de mieux soutenir les doctorantes et doctorants, voire les chercheuses et chercheurs novices dans la rédaction de ce genre de texte. Le concept de genre textuel étant au cœur de notre démarche d'analyse, il constitue le socle de notre cadre conceptuel.

\section{Cadre conceptuel : les caractéristiques d'un genre textuel}

Au vu de la grande diversité des pratiques scripturales dans le milieu académique, que ce soit du côté des étudiantes et étudiants ou des membres du corps professoral, ainsi que de la variété de leurs fonctions (diffusion des connaissances, évaluation, enseignement...), le concept de genre textuel est utile pour catégoriser les différents écrits que devront lire et produire les étudiants et les chercheurs dans leur parcours universitaire. Concept fondamental en didactique de l'écrit, les genres textuels, ou genres du discours, "sont des produits culturels, propres à une société donnée, élaborés au cours de son histoire et relativement stabilisés. Ils régissent les discours des membres de cette société engagés dans les infinies situations de la vie sociale» (Reuter, 2007, p. 117). L'appropriation du concept de genre textuel par les étudiantes et étudiants est une condition pour être en mesure d'agir sur le plan du langage, tant à l'oral qu'à l'écrit, et cela parce que toute communication entre humains s'inscrit dans un genre du discours (Schneuwly et Sales Cordeiro, 2006). Dans la continuité des travaux de Volochinov et Bakhtine ${ }^{3}$ puis de ceux de l'équipe de didactique des langues de Genève (Bronckart, 1996; Dolz, Noverraz et Schneuwly, 2001; Schneuwly et Dolz, 1997), qui mettent au premier plan l'interaction verbale et l'aspect sociodiscursif des productions langagières, le concept de genre textuel a été repris, au Québec, par Chartrand, Émery-Bruneau et Sénéchal (2015) qui le définissent comme suit : « un ensemble de productions langagières orales ou écrites qui, dans une culture donnée, possèdent des caractéristiques communes d'ordres communicationnel, textuel, sémantique, grammatical, graphique ou visuel et/ou d'oralité, souples mais relativement stables dans le temps » (p. 3). Ces didacticiennes retiennent cinq types de caractéristiques dont l'identification permet d'affirmer qu'un texte appartient à une famille spécifique.

- Les caractéristiques communicationnelles concernent la situation et l'intention de communication : qui écrit (énonciateur)? à qui (destinataire)? dans quel but (intention)? d'où (lieu de production)?

2 - Formation et profession 29(3), 2021 
- Les caractéristiques textuelles renvoient à des aspects qui traversent le texte : sa structure générale, le système énonciatif (manifestation de l'énonciateur par l'emploi de pronoms personnels ou de déterminants possessifs, par le phénomène de la modalisation, par la référence aux discours d'autrui), le système des temps verbaux, le type de séquences qui composent le texte (descriptive, narrative, argumentative...).

- Les caractéristiques sémantiques concernent entre autres les aspects liés au type de vocabulaire utilisé dans le texte (familier, courant, soutenu, spécialisé...).

- Les caractéristiques grammaticales renvoient à des particularités comme le type de phrases utilisées ou la ponctuation.

- Enfin, les caractéristiques graphiques ou visuelles concernent l'usage des tableaux, figures, graphiques, images, etc.

Dans notre analyse des articles scientifiques, l'attention a été portée sur l'intention de communication et, particularité de notre recherche, sur les moyens mis en œuvre pour actualiser cette intention de communication, c'est-à-dire que nous avons répertorié les éléments de contenu les plus fréquents permettant d'atteindre le but fixé. Le système énonciatif a également fait l’objet d'une analyse approfondie. Nous avons relevé l'usage des pronoms personnels et des déterminants possessifs ainsi que les modes de référence aux discours d'autrui. À ce sujet, nous nous appuyons sur les catégories proposées par Boch, Grossmann et Rinck (2015) qui distinguent deux modes de référencement aux discours d'autrui, à savoir l'« évocation » et le « discours rapporté » (p. 231). L'évocation consiste à faire référence à des travaux de recherche ou à des chercheurs pour convoquer un concept ou mentionner un phénomène, une idée, un principe, une théorie..., mais sans reformuler des propos. Le discours rapporté quant à lui peut prendre la forme d'une citation, il est alors encadré par des guillemets, ou d'une reformulation, c'est-à-dire que l'énonciateur fait siens les propos rapportés dans un énoncé plus ou moins proche de l'original. Les guillemets ne sont alors pas nécessaires, mais la référence aux chercheurs, avec la date de publication, l'est.

\section{Méthodologie}

Nous nous sommes donné pour objectif d'analyser chaque section de l'article scientifique du domaine des sciences de l'éducation afin de mieux cerner les caractéristiques communicationnelles, textuelles, sémantiques, grammaticales, graphiques et visuelles de ces sections. L'approche méthodologique retenue pour cette recherche descriptive est l'analyse textuelle, proche de l'analyse de discours. Nous présentons ici le corpus de textes analysés et décrivons les critères définis pour le constituer, puis nous explicitons la démarche d'analyse de données réalisée.

\section{Le corpus sélectionné et analysé}

Un modèle d'échantillonnage non probabiliste par choix raisonné (ou intentionnel) a été privilégié afin de sélectionner les articles (Fortin et Gagnon, 2010). Nous avons procédé de façon systématique, car nous voulions un échantillon représentatif de la diversité des articles écrits en français et diffusés récemment dans des revues scientifiques canadiennes du domaine des sciences de l'éducation. Sept revues ont été ciblées pour créer le corpus quantitatif (Tableau 1). 


\section{Tableau 1}

Le corpus d'analyse

\begin{tabular}{lcc}
\hline Noms des revues scientifiques & $\begin{array}{c}\text { Années de publication } \\
\text { des articles }\end{array}$ & $\begin{array}{c}\text { Nombre d'articles } \\
\text { analysés }\end{array}$ \\
\hline Nouveaux cahiers de la recherche en éducation (NCRÉ) & $2015-2017$ & 10 \\
\hline Revue des sciences de l'éducation (RCÉ) & $2015-2017$ & 10 \\
\hline Formation et profession (FP) & 2017 & 10 \\
\hline Revue des sciences de l'éducation de McGill (RSÉM) & $2016-2017$ & 10 \\
\hline Éducation et francophonie (ÉF) & 2017 & 10 \\
\hline Langage et littératie (LL) & 2017 & 10 \\
\hline Revue canadienne de l'éducation (RCÉ) & 2017 & 10 \\
\hline Total & & 70 \\
\hline
\end{tabular}

Les revues sélectionnées répondent aux critères suivants : elles sont canadiennes, elles diffusent des travaux en sciences de l'éducation, elles diffusent des recherches arbitrées par des pairs et elles sont facilement accessibles (libre accès et en ligne). À noter que le statut de l'auteur (professeur, étudiant, stagiaire ou professionnel de recherche) n'a pas été pris en compte dans la sélection, mais soulignons néanmoins que, dans 56 des 70 articles retenus, le premier auteur est une personne ayant le statut de professeur. Ajoutons que 22 articles, soit un peu moins du tiers du corpus, sont écrits par un seul auteur, 24 par deux auteurs, 14 par trois auteurs, et 10 articles par quatre auteurs et plus.

La démarche d'analyse s'est réalisée en deux temps. Nous avons d'abord travaillé de manière inductive à partir d'un corpus initial constitué d'une douzaine d'articles. Lobjectif consistait à dégager les caractéristiques spécifiques de chaque section des articles à partir des régularités observées. Des fiches synthèses de chaque section ont été produites à cette étape, constituant une première série de catégories émergentes. Pour valider cette première analyse de type qualitatif, nous avons dans un deuxième temps procédé à une analyse quantitative sur un corpus élargi $(\mathrm{n}=70)$; cette opération a permis d'éprouver et de préciser les catégories, puis d'établir quelles étaient les caractéristiques, communes et spécifiques, aux sections de l'article scientifique.

Pour constituer le corpus initial $(n=12)$, un ou deux articles du dernier numéro disponible de chaque revue parue en 2017 ont été retenus. Puis, pour constituer le corpus élargi $(n=70)^{4}$, les deux premiers articles parus dans chaque numéro antérieur ont été ajoutés, soit du plus récent au moins récent, jusqu’à ce que soit recueilli un nombre de 10 articles par revue, issus de différents numéros. Ainsi, le corpus comprend des articles publiés entre 2015 et 2017.

\section{Le déroulement de l'étude}

La démarche s'est déroulée en deux phases: inductive d'abord pour dégager les caractéristiques communes et spécifiques à chaque section du corpus initial et stabiliser les sous-catégories; déductive ensuite pour procéder, sur l'ensemble du corpus, à une analyse textuelle proche de l'analyse de discours tout en utilisant les sous-catégories d'analyse préalablement définies. Voici les catégories retenues lors de la première étape, telles que proposées par Chartrand et al. : 
- caractéristiques communicationnelles : intention de communication; destinataire; énonciateur;

- caractéristiques textuelles : structure du texte, système énonciatif, système des temps verbaux, procédés langagiers;

- caractéristiques sémantiques : lexique;

- caractéristiques grammaticales : structure de phrase et ponctuation;

- caractéristiques graphiques et typographiques : tableau, figure, schéma, etc.

À ces catégories s'en ajoute une autre, qui n'apparait pas dans Chartrand et al. (2015), mais qui a émergé lors de l'analyse des intentions de communication : en nous interrogeant sur le but de chacune des sections, nous avons été amenées à nous questionner aussi sur les moyens mis en œuvre pour atteindre ces buts variés. Autrement dit, nous nous sommes demandé comment s'actualise l'intention de communication dans chaque section de l'article scientifique. Cette catégorie, indispensable dans une visée de formation, est la seule qui présente des éléments de contenu propre à l'article scientifique, toutes les autres étant centrées sur des aspects formels de ce genre textuel.

Dans la phase inductive, le traitement section par section a imposé d'exécuter la démarche autant de fois que nécessaire pour tenir compte de l'organisation textuelle variable en matière de regroupement des sections. Par exemple, certains articles sont exempts de la section introduction, ou alors celle-ci est fusionnée avec la problématique; d'autres articles ne comptent qu'une section pour la présentation des résultats et la discussion, etc. En tout, dix configurations ont été relevées : 1 . Résumé; 2 . Introduction; 3. Introduction et problématique; 4. Problématique; 5. Cadre conceptuel, théorique ou de référence; 6. Méthodologie; 7. Résultats; 8. Résultats et discussion; 9. Discussion; 10. Conclusion;

Pour chaque section, la démarche a été la même :

1) dresser des sous-catégories potentielles de la section à partir d'une analyse préliminaire de six textes;

2) analyser systématiquement les caractéristiques de différents ordres dans une douzaine d'articles (exemple de caractéristiques d'ordre textuel : proportion de la section par rapport à l'article; utilisation ou non d'intertitres; présence ou absence de marques énonciatives);

3) ajuster les sous-catégories préétablies à la lumière de l'analyse dans les douze articles du corpus initial;

4) produire des fiches synthèses des caractéristiques de chaque section, sur le modèle des fiches de Chartrand, Émery-Bruneau et Sénéchal (2015);

5) analyser les 70 textes du corpus;

6) valider les fiches synthèses ;

7) établir des constats ou généralisation sur les caractéristiques génériques de chaque section. 


\section{Outils d'analyse des données}

Différents outils et logiciels ont été utilisés pour faciliter le traitement et l'analyse des données. D'abord, tous les articles (initialement en format PDF) ont été convertis en format Word afin de compter le nombre de mots (total et par section, excluant les références bibliographiques) à l'aide de l'outil statistique que l'on retrouve sous l'onglet « Révision » dans ce logiciel de traitement de texte.

Les 70 articles ont ensuite été transférés dans le logiciel N’Vivo 11 pour être analysés à partir des catégories suivantes :

- Section (résumé, introduction, problématique, etc.);

- Intention de communication (différentes sous-catégories selon les contenus utilisés pour mettre en œuvre l'intention de communication);

- Système énonciatif (pronoms personnels à la première personne du pluriel ou du singulier, pronoms possessifs);

- Mode de référence aux discours d'autrui (évocation et reformulation ${ }^{5}$; citation)

- Structure du texte (titres et intertitres; séquences introductives); marques graphiques (tableaux, figures, graphiques);

- Informations sur les auteurs (nombre, statut, université d'attache).

L'annexe 2 présente les catégories et les variables observées sur le corpus élargi pour l'analyse de contenu avec N'Vivo 11.

Dans la section suivante sont exposés une partie des résultats de l'analyse quantitative des 70 articles réalisée avec la grille (annexe 1).

\section{Résultats : caractéristiques de l'article scientifique en sciences de l'éducation}

Parmi les résultats foisonnants de l'analyse, nous retenons, pour cette contribution, ceux qui mettent en évidence les distinctions entre chacune des sections de l'article scientifique sur le plan des caractéristiques communicationnelles et textuelles, faisant de celui-ci un écrit d'apparence uniforme, mais pourtant constitué de parties ayant des caractéristiques particulières. Nous commençons par présenter l'intention de communication générale de l'article, et les intentions de communication de chaque section. Nous traitons ensuite des caractéristiques textuelles propres à chaque section : la structure du texte et le système énonciatif. Enfin, nous présentons des éléments de contenu choisis pour actualiser l'intention de communication dans chacune des sections.

\section{Les intentions de communication}

Contribuer à l'avancement des connaissances scientifiques, telle est généralement l'intention de communication globale du genre textuel « article scientifique ». Pour mettre en œuvre cette intention, l'énonciateur introduit généralement son objet de recherche, le problématise, établit un cadre conceptuel, décrit la méthodologie qu'il a élaborée pour mener les travaux, puis présente les résultats 
de sa recherche, en discute et conclut son article. Et chacune de ces sections se construit autour d'une intention de communication qui lui est propre :

\begin{tabular}{|l|l|}
\hline \multicolumn{1}{|c|}{ Sections } & \multicolumn{1}{c|}{ Intentions de communication } \\
\hline Résumé & Présenter les grandes lignes de la recherche \\
\hline Introduction & $\begin{array}{l}\text { Donner au destinataire une idée générale de la recherche pour susciter l'intérêt et faciliter la compré- } \\
\text { hension de l'article }\end{array}$ \\
\hline Problématique & Justifier la pertinence sociale et/ou scientifique de s'intéresser au problème choisi \\
\hline Cadre conceptuel & Situer les choix théoriques et épistémologiques dans laquelle la recherche a été réalisée \\
\hline Méthodologie & Expliquer comment la recherche a été réalisée et montrer qu'elle a été menée avec rigueur \\
\hline Résultats & Présenter les résultats marquants \\
\hline Discussion & Montrer ce que nous apprennent les résultats (par rapport à d'autres recherches) \\
\hline Conclusion & Rappeler les éléments clés de la recherche et souligner ses apports et prolongements \\
\hline
\end{tabular}

La variabilité dans l'intention de communication pour chaque section se répercute sur certains aspects du texte tels que la structure et la longueur des sections, la posture adoptée par l'énonciateur (il se manifeste plus ou moins selon les sections), le mode de référence au discours d'autrui, les temps verbaux, etc.

Dans notre corpus, sur le plan de la structure, les sections des articles scientifiques ne sont pas découpées de la même façon d'un article à l'autre. Sur les 70 articles, 13 sont "standards »" , c'est-à-dire qu'ils comprennent, dans l'ordre et de manière exclusive, les sections suivantes : résumé, introduction, problématique, cadre conceptuel, méthodologie, résultats, discussion et conclusion. Dans les autres articles, on observe des configurations variables (tableau 2).

\section{Tableau 2}

Composition du corpus

\begin{tabular}{lrr}
\hline & Total & Moyenne $^{7}$ \\
\hline Standard & $13 / 70$ & $18,6 \%$ \\
Sans introduction & $12 / 70$ & $17,1 \%$ \\
\hline Sans problématique & $15 / 70$ & $21,4 \%$ \\
Sans cadre conceptuel & $5 / 70$ & $7,1 \%$ \\
\hline Sans méthodologie & $7 / 70$ & $10,0 \%$ \\
Sans résultats & $9 / 70$ & $12,9 \%$ \\
\hline Sans discussion & $4 / 70$ & $5,7 \%$ \\
Sans conclusion & $5 / 70$ & $7,1 \%$ \\
\hline Introduction combinée à la problématique & $12 / 70$ & $17,1 \%$ \\
Introduction combinée au cadre conceptuel & $1 / 70$ & $1,4 \%$ \\
\hline Problématique combinée au cadre conceptuel & $5 / 70$ & $7,1 \%$ \\
Méthodologie combinée aux résultats & $1 / 70$ & $1,4 \%$ \\
\hline Résultats combinés à la discussion & $9 / 70$ & $12,9 \%$ \\
\hline Discussion combinée à la conclusion & $5 / 70$ & $7,1 \%$ \\
\hline
\end{tabular}


À partir des 13 articles standards, nous avons établi la proportion occupée par chaque section par rapport à l'ensemble de l'article (excluant la bibliographie) (Figure 2).

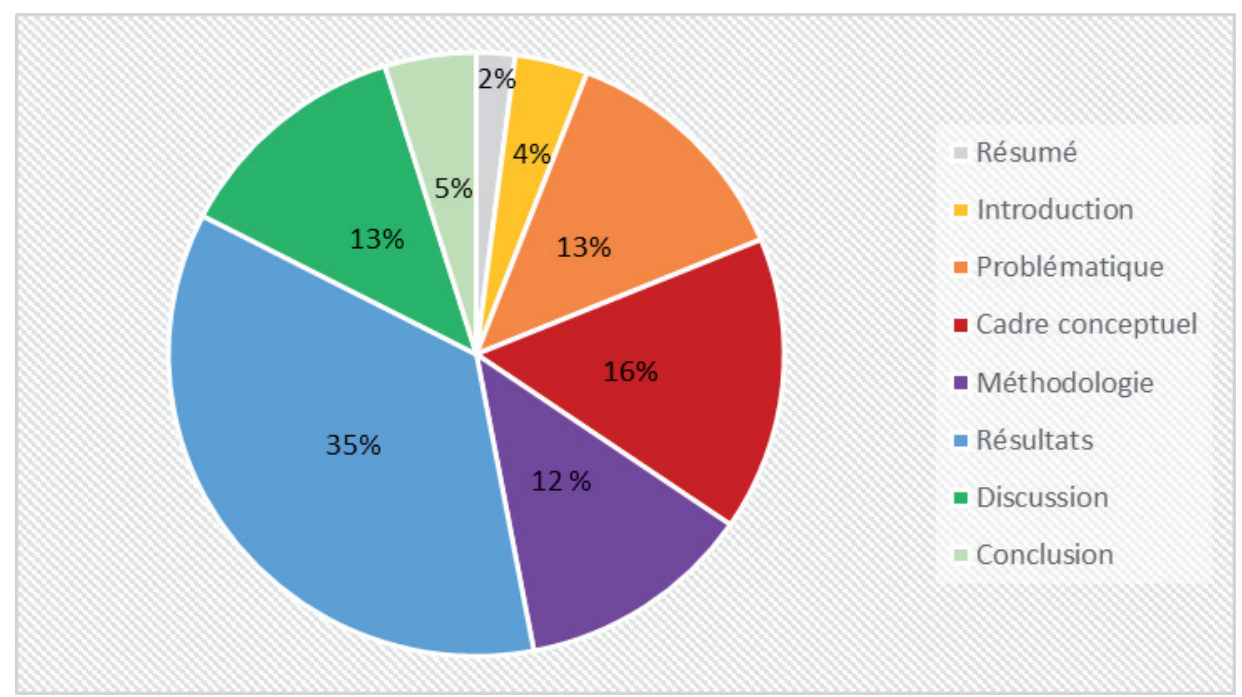

\section{Figure 2}

Proportion des sections à l'intérieur de l'article scientifique

Il ressort que l'introduction et la conclusion ont un ratio à peu près semblable, de l'ordre $4 \%$ et $5 \%$. La problématique (13\%), le cadre conceptuel (16\%), la méthodologie (12\%) et la discussion (13\%) sont aussi de longueur presque équivalente tandis que la section des résultats se distingue nettement en occupant en moyenne $35 \%$ de la longueur totale (sans les références bibliographiques), soit environ le tiers de l'article.

\section{Le système énonciatif}

Au sujet du système énonciatif, dans cet article, nous nous intéressons à l'emploi des pronoms personnels et des déterminants possessifs, ainsi qu'aux références aux discours d'autrui.

Le corpus compte un seul article contenant le pronom personnel à la première personne du singulier (je), et cela même si 20 des 70 articles sont rédigés par un seul auteur. En revanche, le pronom personnel à la première personne du pluriel «nous » se trouve dans 54 articles et le pronom indéfini « on » dans 29 articles $^{8}$; les déterminants possessifs (mon, notre, nos, etc.) sont présents dans 44 articles.

En ce qui a trait à l'utilisation de ces termes, elle varie selon les différentes sections des 70 articles (figure 4), autrement dit, l'énonciateur ne se manifeste pas avec la même intensité dans chaque section; la figure 4 présente la moyenne ${ }^{9}$ de toutes les occurrences de ces déterminants possessifs, ainsi que les pronoms personnels et indéfinis, lesquels sont un indice parmi d'autres de la présence de l'auteur dans son texte. 


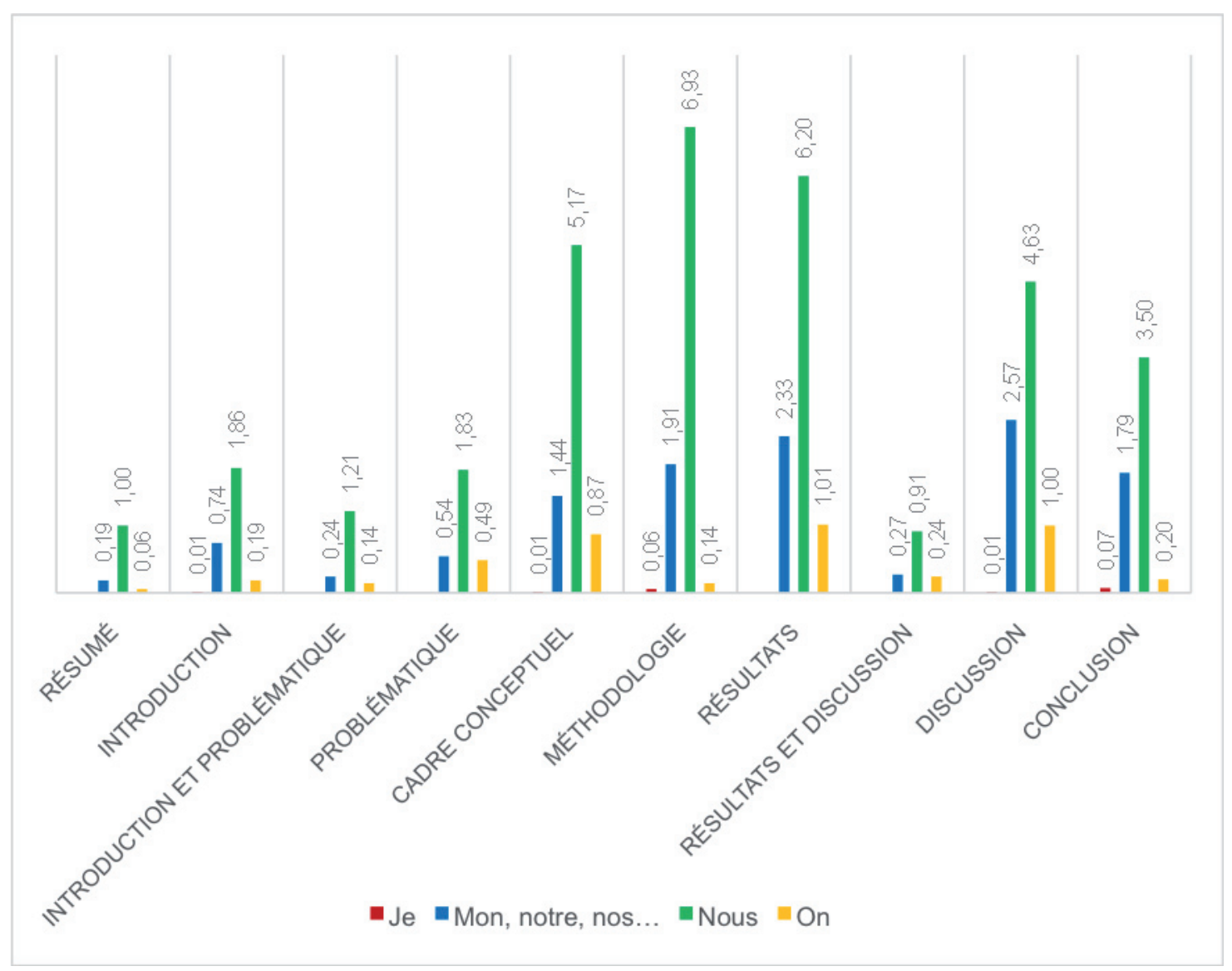

\section{Figure 4}

Moyenne des occurrences des déterminants possessifs et des pronoms personnels et indéfinis par section

De cette figure, il ressort que :

- le pronom personnel à la première personne du pluriel « nous » est utilisé dans toutes les sections, son emploi est toutefois plus important dans le cadre conceptuel $(5,17)$, la méthodologie $(6,93)$ et les résultats $(6,20)$;

- le pronom indéfini « on » est utilisé dans toutes les sections, il est principalement utilisé dans le cadre conceptuel $(0,87)$, les résultats $(1,01)$ et la discussion $(1,00)$;

- les déterminants possessifs (mon, notre, nos, etc.) sont également utilisés dans toutes les sections, leur emploi est plus marqué dans les résultats $(2,33)$ et la discussion $(2,57)$.

Rappelons que le système énonciatif inclut les "modes de référence au discours d'autrui » (Boch, Grossman et Rinck, 2015, p. 233). En nous appuyant sur la typologie établie par ces chercheurs, nous observons que, dans le corpus analysé, la citation est présente dans 21 des 70 articles tandis que l'évocation ${ }^{10}$ et la reformulation sont présentes dans 60 articles. La moyenne du nombre des références au discours d'autrui a été relevée par section dans les 70 articles (figure 5). De manière générale, l'évocation et la reformulation sont nettement plus souvent utilisées que la citation, surtout dans les 
sections comme le cadre conceptuel, la problématique et la discussion. Le cadre conceptuel est la section où sont le plus souvent utilisées la citation $(2,84)$ et l'évocation ou la reformulation $(27,56)$, notamment en raison des notions, concepts ou modèles théoriques empruntés à d'autres chercheurs et décrits en fonction des objectifs de la recherche faisant l'objet de l'article.

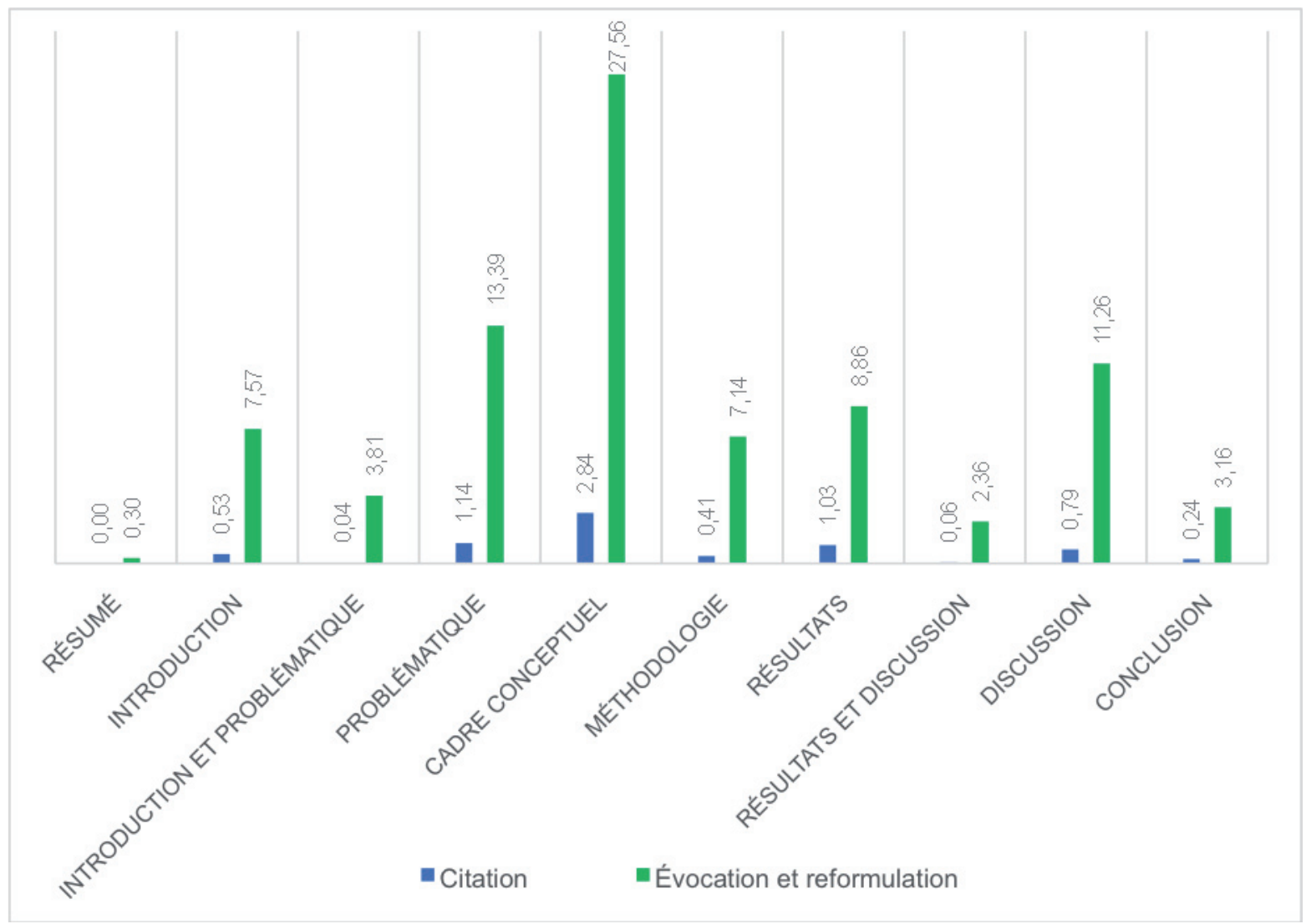

Figure 5

Moyenne des occurrences des modes de référence au discours d'autrui

\section{Mise en œuvre de l'intention de communication}

La figure 6 montre les différents moyens utilisés pour mettre en œuvre l'intention de communication en fonction de leur occurrence par section. 


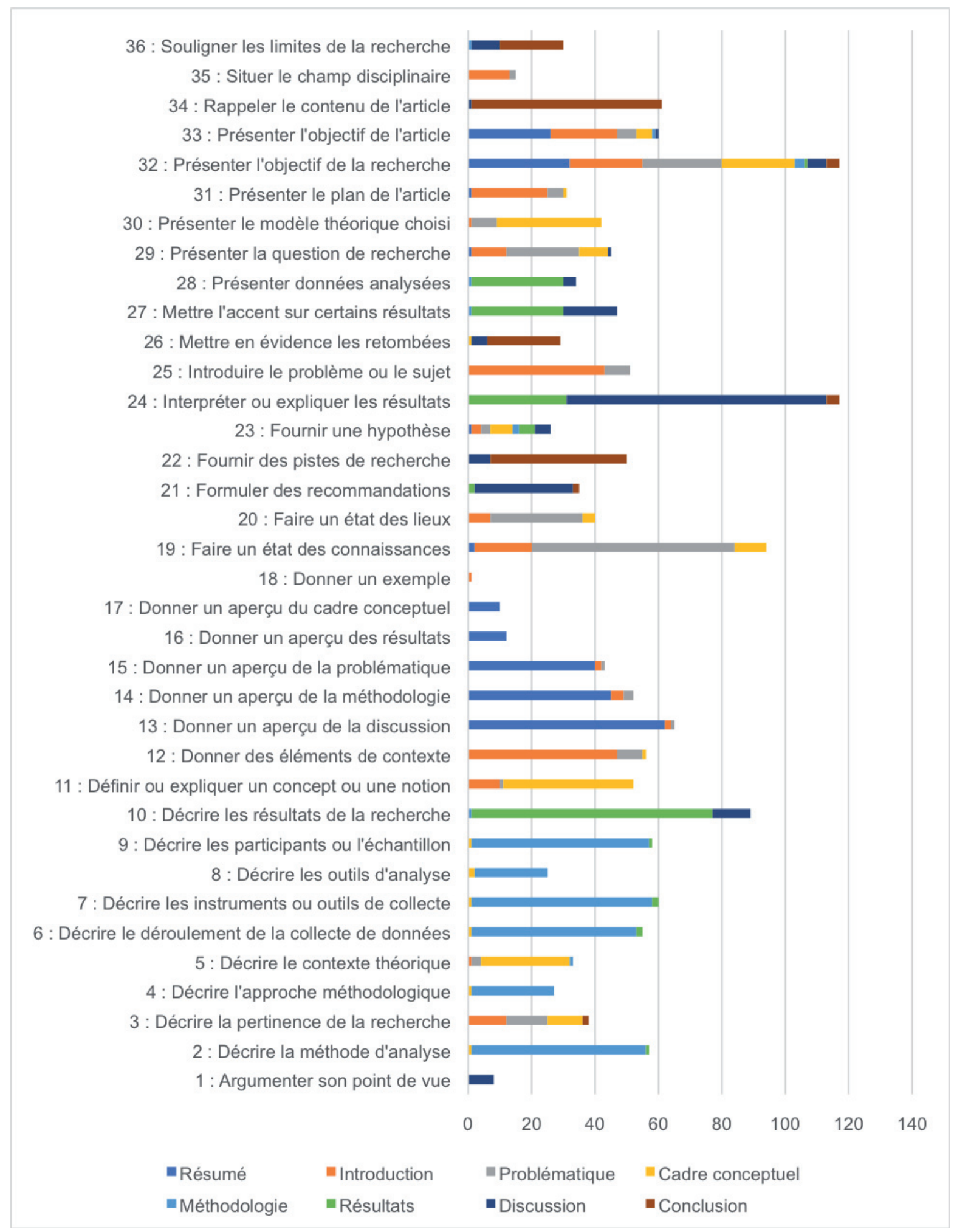

\section{Figure 6}

Occurrence des moyens utilisés pour mettre en cuvre l'intention de communication par section 
Nous constatons que, bien qu'il arrive que des moyens apparaissent dans deux sections, par exemple « donner des éléments du contexte » (no 12), qui se trouve à la fois dans les sections «introduction » et "problématique», ou "fournir des pistes de recherche » (no 22), qui apparait à la fois dans les sections « discussion » et " conclusion », une distinction s'observe dans la mesure où il s'agit rarement de plus d'une dizaine d'articles, sur les 70 du corpus, qui sont concernés par ce chevauchement entre deux sections. Par conséquent, des régularités génériques marquées nous permettent de caractériser les sections en fonction des moyens de mettre en œuvre les intentions de communication.

En revanche, nous observons quatre moyens, parmi les 36 identifiés, qui reviennent dans au moins trois sections : "décrire la pertinence de la recherche » (no 3), « fournir une hypothèse » (no 23), " présenter la question de recherche » (no 29) et " présenter l'objectif de la recherche » (no 32), soit des aspects centraux d'une recherche qui sont soulignés plus d'une fois dans un article.

En résumé, les principaux moyens d'actualiser les intentions de communication identifiés pour chaque section sont les suivants (présentés par ordre de fréquence observée dans le corpus des 70 articles scientifiques) :

\begin{tabular}{|c|c|}
\hline Sections & Mise en œuvre des intentions de communication \\
\hline Résumé & $\begin{array}{ll}\text { - } & \text { Donner un aperçu de la problématique } \\
\text { - } & \text { Donner un aperçu de la méthodologie } \\
\text { - } & \text { Donner un aperçu de la discussion } \\
\text { - } & \text { Présenter l'objectif de la recherche } \\
\end{array}$ \\
\hline Introduction & $\begin{array}{ll}\text { - } & \text { Donner des éléments du contexte } \\
\text { - } & \text { Décrire le problème ou le sujet de la recherche } \\
\text { - } & \text { Présenter le plan de l'article } \\
\text { - } & \text { Présenter l'objectif de la recherche } \\
\text { - } & \text { Présenter l'objectif de l'article }\end{array}$ \\
\hline Problématique & $\begin{array}{ll}\text { - } & \text { Faire un état des connaissances (recension des écrits) } \\
\text { - } & \text { Présenter la question de recherche } \\
\text { - } & \text { Présenter l'objectif (ou les objectifs) de la recherche }\end{array}$ \\
\hline Cadre conceptuel & $\begin{array}{ll}\text { - } & \text { Définir ou expliquer un concept ou une notion } \\
\text { - } & \text { Présenter le modèle théorique } \\
\text { - } & \text { Décrire le contexte théorique } \\
\text { - } & \text { Présenter l'objectif de la recherche } \\
\end{array}$ \\
\hline Méthodologie & $\begin{array}{ll} & \text { Décrire les participants ou l'échantillon } \\
\text { - } & \text { Décrire la méthode d'analyse des données } \\
\text { - } & \text { Décrire les instruments ou outils de collecte des données } \\
\text { - } & \text { Décrire le déroulement de la collecte de données } \\
\text { - } & \text { Décrire l'approche méthodologique } \\
\text { - } & \text { Décrire les outils d'analyse }\end{array}$ \\
\hline Résultats & $\begin{array}{l}\text { - } \quad \text { Décrire les instruments ou outils de collecte des données } \\
\text { - } \quad \text { Présenter les données analysées } \\
\text { - } \quad \text { Mettre l'accent sur certains résultats } \\
\text { - } \quad \text { Interpréter ou expliquer les résultats } \\
\text { - } \quad \text { Présenter les données analysées et mettre en évidence celles qui permettent de répondre aux } \\
\quad \text { questions de la recherche, de valider les hypothèses ou d'atteindre les objectifs de la recherche } \\
\text { - } \quad \text { Décrire les résultats de la recherche de façon synthétique }\end{array}$ \\
\hline Discussion & $\begin{array}{l}\text { - } \quad \text { Reprendre les résultats les plus importants, les expliquer et les interpréter à la lumière du cadre } \\
\text { conceptuel de la recherche et/ou d'autres résultats de recherches } \\
\text { - } \quad \text { Formuler des propositions ou recommandations } \\
\text { _ } \quad \text { Souligner les limites de la recherche }\end{array}$ \\
\hline
\end{tabular}


L'écriture scientifique se transforme. Dans notre démarche, nous avons voulu mettre en évidence, à travers la description de ses diverses caractéristiques, que l'article scientifique peut être abordé comme la somme de ses parties plutôt que comme un bloc monolithique.

\section{Discussion conclusive}

Au terme de cette démarche d'analyse d'un corpus d'articles scientifiques, il est important de rappeler que les résultats présentés dans cet article décrivent l'article scientifique du domaine des sciences de l'éducation, dans la francophonie contemporaine (2015 à 2017). Autrement dit, tel que défini par Chartrand et al. (2015), le genre textuel s'inscrit «dans une culture donnée » et il possède des caractéristiques « souples mais relativement stables dans le temps ». Dans le contexte qui est le nôtre, le genre textuel en question - l'article scientifique - est marqué par la culture scientifique du domaine des sciences de l'éducation en contexte francophone. Une investigation plus poussée serait nécessaire pour établir dans quelle mesure la formation des chercheurs et chercheuses diplômés d'universités des quatre coins du monde, ainsi que des normes éditoriales plus conformistes dans certaines revues, teintent les écrits scientifiques, car en cette ère de mondialisation de la culture scientifique, on peut penser que les écrits tendent plutôt vers l'uniformisation, compte tenu du caractère international des équipes de recherche et des comités d'évaluation par les pairs, de la mobilité étudiante... Avantage, inconvénient? Assurément, on ne peut que se réjouir de l'accès de plus en plus libre aux écrits scientifiques, car cela permet aux chercheurs et chercheuses de tous les horizons de participer à la construction des connaissances scientifiques, mais cela va de pair avec des contraintes établies par une communauté scientifique dominante au détriment, peut-être, d'autres formes de recherches, de pensées, d'écriture.

On pourrait investiguer davantage aussi pour établir la nature de l'évolution des écrits scientifiques dans le temps, en particulier sur le plan du référencement au discours d'autrui. L'accès facilité aux écrits scientifiques a largement accentué les stratégies de copier-coller, de créacollage (Peters et Gervais, 2016; Peters, 2015) ou de " patchwriting " (Beaudet, 2015). Mal maitrisées, ces stratégies d'écriture peuvent conduire au plagiat, mais entre les mains de scripteurs avertis, elles mènent à la production de nouveaux écrits abondamment référencés.

En terminant, mentionnons que nous utilisons les résultats de cette recherche dans nos pratiques enseignantes, que ce soit dans les cours que nous offrons aux différents cycles universitaires ou sous forme d'ateliers animés dans des contextes variés (Blaser et Lanctôt, 2021; Blaser, Émery-Bruneau et Lanctôt, 2019). Par exemple, la connaissance fine des caractéristiques de l'article scientifique permet de bien distinguer ce genre textuel de l'article professionnel, un genre qui fait aussi partie des écrits que sont amenés à lire et à produire les étudiantes et étudiants des cycles supérieurs et les chercheurs et chercheuses. Ces deux genres de texte peuvent avoir de nombreux points communs, mais ils se distinguent clairement sur le plan des caractéristiques communicationnelles, entre autres par leurs destinataires respectifs : l'article scientifique est destiné à un public restreint, la communauté scientifique à laquelle appartient le chercheur ou la chercheuse, tandis que l'article professionnel est destiné à un public de personnes professionnelles intervenant souvent dans les milieux de pratique. L'intention de communication est aussi un critère permettant de distinguer les deux genres d'articles : le premier vise généralement à contribuer à l'avancement des connaissances scientifiques (et il est soumis à une évaluation par les pairs) tandis que le second peut avoir des intentions variées comme 
présenter l'état d'une situation ou d'un phénomène, faire des recommandations pour inciter à agir, dénoncer une situation, etc. (il n'est pas soumis à une évaluation par les pairs).

L'entrainement à observer les caractéristiques des textes lus et produits dans la sphère universitaire - et ailleurs! - a pour effet de développer un regard plus vigilent et critique sur le monde de l'écrit, ce qui n’est pas la moindre des qualités dans le domaine de la recherche.

\section{Notes}

1 C'est le cas par exemple à l'Université Laval (DID7000 - Analyse et écriture de textes de genre universitaire) ou à l'Université de Sherbrooke dans le cadre du programme de Doctorat professionnel (DED903 - Lire et écrire pour mobiliser la recherche au profit de l'intervention).

2 Nous entendons par section, les grandes parties qui organisent le plus souvent un article scientifique, soit la problématique, le cadre conceptuel, la méthodologie, etc.

3 Pour des précisions sur le débat concernant l'origine du concept de genre, voir l'article de Bota et Bronckart (2007)

4 Le corpus quantitatif $(\mathrm{n}=70)$ inclut les articles du corpus initial.

5 Nous avons fait le choix, dans ce contexte, de ne pas distinguer les phénomènes d'évocation et de reformulation, même si nous sommes conscientes qu'ils ne sont pas de même nature. Nous avons plutôt retenu l'emploi des guillemets comme critère de distinction des modes de référence au discours d'autrui. Deux cas de figure se présentent donc : un mode de référence au discours d'autrui encadré de guillemets (= citation); un mode de référence au discours d'autrui non encadré de guillemets (= évocation ou reformulation).

6 Le mot «standard » a été retenu à défaut de trouver mieux pour désigner les articles qui comportent toutes les sections sans chevauchement entre les unes et les autres. Le reste des articles a été classé dans la catégorie « non-standards » pour deux raisons : une ou plusieurs sections sont absentes dans l'article ou deux sections ou plus y sont combinées.

7 Moyenne arrondie à la décimale.

8 De manière non exclusive. Autrement dit, dans certains articles, les pronoms « nous » et « on » sont présents en même temps.

9 Pour arriver à ces moyennes, nous avons d'abord additionné toutes les occurrences des 70 articles (par exemple, nous avons relevé 485 « nous » dans la section « méthodologie »), puis nous avons divisé ce total par le nombre d'articles concernés.

10 Lévocation consiste à faire référence à des chercheurs, mais sans citer ou reformuler leurs propos.

\section{Références}

Beaudet, C. (2015). Littéracie universitaire, patchwriting et impéritie. Le français aujourd'bui(190), 99-112.

Belleville, G. (2014). Assieds-toi et écris ta thèse! Trucs pratiques et motivationnels. Québec: PUL.

Blaser, C. et Lanctôt, S. (2021). Accompagner les doctorantes et doctorants dans l'appropriation de genres textuels : connaitre les maladresses ou erreurs scripturales relatives à la définition d'un concept. In M.-H. Forget et A. Malo (Dir.), (Se) Former à et par l'écriture du qualitatif (pp. 31-53). Québec: PUL.

Blaser, C., Émery-Bruneau, J. et Lanctôt, S. (2019). Enseigner le concept de genre textuel à l'université pour outiller les étudiants à mieux lire et écrire. Formation et pratiques d'enseignement en questions(25),103-116.

Boch, F. (2013). Former les doctorants à l'écriture de la thèse en exploitant les études descriptives de l'écrit scientifique. Linguagem em (Dis)curso, 13(3), 543-568.

Boch, F., et Frier, C. (2015). Ecrire dans l'enseignement supérieur, des apports de la recherche aux outils pédagogiques: ELLUG, coll. Didaskein. 
Boch, F., Cavalla, C., Pétillon, S. et Rinck, F. (2015). Travailler le texte : ponctuation, anaphores et collocations. In F. Boch et C. Frier (Dir.), Ecrire dans l'enseignement supérieur, des apports de la recherche aux outils pédagogiques (pp. 194-228): ELLUG, coll. Didaskein.

Boch, F., Grossmann, F. et Rinck, F. (2015). Écrire en tant qu'apprenti-chercheur. In F. Boch et C. Frier (dir.), Écrire dans l'enseignement supérieur, des apports de la recherche aux outils pédagogiques (pp. 211-247): ELLUG, coll. Didaskein.

Bota, C. et Bronckart, J.-P. (2007). Volochinov et Bakhtine : deux approches radicalement opposées des genres de texte et de leur statut. $\operatorname{Lin} x,(56)$, p. 67-83

Bronckart, J.-P. (1996). Activité langagière, textes et discours: Pour un interactionisme socio-discursif. Delachaux et Niestlé.

Chartrand, S.-G., Émery-Bruneau, J., et Sénéchal, K. (2015). Caractéristiques de 50 genres pour développer les compétences langagières en français (Didactica, c.é.f.).

Delcambre, I., et Lahanier-Reuter, D. (2010). Les littéracies universitaires. Influence des disciplines et du niveau d'étude dans les pratiques de l'écrit. In C. Blaser et M.-C. Pollet, L'appropriation des écrits universitaires (p. 11-42). Presses universitaires de Namur.

Delcambre, I., et Lahanier-Reuter, D. (2012). Littéracies universitaires: Présentation. Pratiques, 153-154, 3-19.

Delcambre, I., et Pollet, M.-C. (2014). Présentation. Revue de recherches en éducation, 53, 3-8.

Dolz, J., Noverraz, M., et Schneuwly, B. (2001). S'exprimer en français: Séquences didactiques pour l'oral et pour l'écrit (Vol. 4). De Boeck.

Donahue, C. (2008). Écrire à l'université: Analyse comparée en France et aux États-Unis. Presses universitaires du Septentrion.

Donahue, C. (2010). Évolution des pratiques et des discours sur l'écrit à l'université: Étude de cas. Lidil, 41, 137-160.

Lafont-Terranova, J., Niwese, M., et Colin, D. (2016). Développer des dispositifs d'acculturation à l'écriture de recherche: Un enjeu didactique et épistémologique. Pratiques. Linguistique, littérature, didactique, 171-172. http://journals. openedition.org.ezproxy.usherbrooke.ca/pratiques/3201

Pollet, M.-C. (2001). Pour une didactique des discours universitaires: Étudiants et systèmes de communication à l'université. De Boeck université.

Pollet, M.-C. (2004). Appropriation et écriture de savoirs chez des étudiants de première année. Une voie difficile entre stockage et élaboration. Pratiques, 121-122, 81-92.

Pollet, M.-C. (2014). L'écrit scientifique à l'aune des littéracies universitaires. Approches théoriques et pratiques. Presses universitaires de Namur.

Pollet, M.-C., Glorieux, C., et Toungouz, K. (2010). Pour un continuum dans l'appropriation d'une littéracie universitaire. In C. Blaser et M.-C. Pollet (Dir.), L'appropriation des écrits universitaires (p. 61-92). Presses universitaires de Namur.

Reuter, Y. (2007). Statut et usages de la notion de genre en didactique(s). Le français aujourd'bui, 159, 11-18.

Scheepers, C., Donahue, C. et Bautier, É. (2021). Former à l'écrit, former par l'écrit dans le supérieur. DeBoeck

Schneuwly, B., et Dolz, J. (1997). Les genres scolaires. Des pratiques langagières aux objets d'enseignement. Repères, 15, 27-41.

Schneuwly, B., et Sales Cordeiro, G. (2006). Le genre de texte comme objet autonome d'enseignement: Comparaison de deux approches didactiques. In G. Sales Cordeiro et D. Vrydaghs (Dir.), Statuts des genres en didactique du français: Recherche, formation et pratiques enseignantes (p. 83-128). Presses universitaires de Namur.

Thyrion, F. (2011). Les voies du discours. Recherche en sciences du langage et en didactique du français. Presses Universitaires de Louvain.

\section{Pour citer cet aritcle}

Blaser, C., Émery-Bruneau, J. et Lanctôt, S. (2021). L'article scientifique en sciences de l'éducation : un genre textuel à l'apparence uniforme constitué de sections aux caractéristiques distinctes. Formation et profession, 29 (3), 1-16. http://dx.doi.org/10.18162/fp.2021.548 


\section{Annexe 1}

Catégories et variables observées sur le corpus quantitatif

\begin{tabular}{|c|c|c|}
\hline Catégories & Variables observées & \\
\hline Sections & $\begin{array}{l}1: \text { Résumé } \\
2: \text { Introduction } \\
3: \text { Introduction et problématique } \\
4: \text { Problématique } \\
5: \text { Cadre conceptuel, théorique ou de référence }\end{array}$ & $\begin{array}{l}6: \text { Méthodologie } \\
7: \text { Résultats } \\
8: \text { Résultats et discussion } \\
9: \text { Discussion } \\
10: \text { Conclusion }\end{array}$ \\
\hline $\begin{array}{l}\text { Mise en œuvre } \\
\text { de l'intention de } \\
\text { communication dans } \\
\text { chaque section }\end{array}$ & $\begin{array}{l}1: \text { Argumenter son point de vue } \\
2: \text { Décrire la méthode d'analyse } \\
3: \text { Décrire la pertinence de la recherche } \\
4: \text { Décrire l'approche méthodologique } \\
5: \text { Décrire le contexte théorique } \\
6: \text { Décrire le déroulement de la collecte de } \\
\text { données } \\
7: \text { Décrire les instruments ou outils de collecte } \\
8: \text { Décrire les outils d'analyse } \\
9: \text { Décrire les participants ou l'échantillon } \\
10: \text { Décrire les résultats de la recherche } \\
11: \text { Définir ou expliquer un concept ou une } \\
\text { notion } \\
12: \text { Donner des éléments de contexte } \\
13: \text { Donner un aperçu de la discussion } \\
14: \text { Donner un aperçu de la méthodologie } \\
15: \text { Donner un aperçu de la problématique } \\
16: \text { Donner un aperçu des résultats } \\
17: \text { Donner un aperçu du cadre conceptuel } \\
18: \text { Donner un exemple }\end{array}$ & $\begin{array}{l}19 \text { : Faire un état des connaissances } \\
20 \text { : Faire un état des lieux } \\
21 \text { : Formuler des recommandations } \\
22 \text { : Fournir des pistes de recherche } \\
23 \text { : Fournir une hypothèse } \\
24 \text { : Interpréter ou expliquer les résultats } \\
25 \text { : Introduire le problème ou le sujet } \\
26 \text { : Mettre en évidence les retombées } \\
27 \text { : Mettre l'accent sur certains résultats } \\
28 \text { : Présenter données analysées } \\
29 \text { : Présenter la question de recherche } \\
30 \text { : Présenter le modèle théorique choisi } \\
31 \text { : Présenter le plan de l'article } \\
32 \text { : Présenter l'objectif de la recherche } \\
33 \text { : Présenter l'objectif de l'article } \\
34 \text { : Rappeler le contenu de l'article } \\
35 \text { : Situer le champ disciplinaire } \\
36 \text { : Souligner les limites de la recherche }\end{array}$ \\
\hline Système énonciatif & $\begin{array}{l}1: \text { Pronoms personnels « je » et « nous » } \\
2: \text { Pronom indéfini « on » }\end{array}$ & $\begin{array}{l}3 \text { : Déterminants possessifs (mon, notre, nos...) } \\
4: \text { Discours rapporté direct et indirect }\end{array}$ \\
\hline Structure du texte & $1:$ Titres et sous-titres & 2: Phrases ou paragraphes introductifs \\
\hline Marques graphiques & $\begin{array}{l}1: \text { Présence de tableaux } \\
2: \text { Présence de figures }\end{array}$ & $3:$ Présence de graphiques \\
\hline Auteurs & $\begin{array}{l}1: \text { Nombre d'auteurs } \\
2: \text { Statut du premier auteur : professeur/ } \\
\text { chercheur ou étudiant }\end{array}$ & $3:$ Université d'attache du premier auteur \\
\hline
\end{tabular}

